\title{
Aspekte der Qualitätssicherung in der operativen Gynäkologie aus der Sicht der Deutschen Gesellschaft für Gynäkologie und Geburtshilfe
}

H. G. Bender

Qualitätskontrolle und -sicherung bzw. -verbesserung scheint eine Entdeckung der letzten Jahre zu sein. Operative Tätigkeit sollte und ist sicher überwiegend durch Qualitätsbewusstsein gekennzeichnet. Was sich gewandelt hat, sind die Aspekte, von welcher Seite und mit welchen Zielen im Grundsatz berechtigte Qualitätsanforderungen an die operative Gynäkologie herangetragen werden. Unter den heutigen Bedingungen unseres Gesundheitswesens ist fast jede programmatische Aktivität - wie auch immer tituliert - letztendlich darauf ausgelegt, eine Kostenersparnis herbeizuführen. Qualität ist ein ohne Frage einforderbares Merkmal, und niemand wird sich einer sinnvollen Kosteneinsparung widersetzen. Fraglich bleibt jedoch, ob Erfassungssysteme alle Tatbestände so detailliert integrieren, dass sich ein Realitäts-widerspiegelndes Bild ergibt und sich nicht in der konkreten Handlung aufgrund insuffizienter Daten Fehlentwicklungen bemerkbar machen, die dann schon zu Schäden geführt haben und schwierig korrigierbar sind.

Die Definition der Qualität in der operativen Gynäkologie drückt sich aus in der Zufriedenheit der operierten Patientin auf der Basis von langfristig optimalen Heilungsergebnissen bei minimaler Belastung durch Nebenwirkungen und Komplikationen in einem möglichst positiven Umfeld. Ein in dieser Definition enthaltenes wesentliches Merkmal ist die Rate und der Schweregrad von operationsbedingten Komplikationen. Die Bemühung um die Minimierung und Beachtung von Komplikationen hat die Bewertung von Operateuren und Operationsverfahren nachhaltig mitgeprägt. Gegenüber den Verbesserungen in der indikationsbestimmenden Problematik wird gegenbilanziert, welche Nebenwirkungs- und Komplikationsquoten $\mathrm{zu}$ bedenken sind und letztendlich auch mit der Patientin vor der Operation angesprochen werden. Nicht zuletzt aufgrund der vorbildhaften Erfahrun- gen aus den Erhebungen zur Perinatologie lag es nahe, dass man sich innerhalb der Deutschen Gesellschaft für Gynäkologie und Geburtshilfe - nicht zuletzt um vergleichbare Bewertungsebenen zu erhalten - um systematische Erhebungen bemüht hat. Darüber wird in anderen Kapiteln dieses Bandes berichtet. Eine wirklich aussagefähige Datenerhebung zur operativen Gynäkologie ist sehr aufwendig. Zunächst einmal erfordern die erfreulicherweise sehr niedrigen Komplikationsraten sehr große Kollektive für eine statistisch relevante Beurteilung der Basis-Komplikationsraten, die verfahrensimmanent unvermeidbar sind und solche, die eine Abweichung aus nicht beeinflussbaren Gründen darstellen oder die korrekturfähig wären und im Zusammenhang damit vermindert oder vermieden werden sollten. Unter diesen Aspekten sind derzeit valide Zahlen nur sehr schwer zu erhalten. Sie können nur aus Daten abgeleitet werden, die repräsentativ für die derzeitige Versorgungslage in den zu beurteilenden Regionen ist. Diesem Anspruch werden die bisher vorliegenden und zumeist herangezogenen Veröffentlichungen nicht gerecht. Muss man - zum Beispiel als Gutachter - Angaben zu Komplikationsraten machen, beruft man sich im Allgemeinen auf die aktuelle Literatur. Deren Relevanz ist jedoch durchaus daraufhin zu hinterfragen, ob nicht eine Selektion zu Zahlenangaben führt, die nicht der Situation in der allgemeinen operativ-gynäkologischen Versorgung in Deutschland entsprechen. Es besteht eine gewisse Wahrscheinlichkeit, dass eine Institution oder eine Person eher geneigt ist, Ergebnisse zu publizieren, wenn sie eher günstiger als im Allgemeinen Durchschnitt sind. Ungünstigere Ergebnisse, die eine Institution oder Person möglicherweise in Erklärungsschwierigkeiten bringen könnten, dürften eher weniger Anlass für eine Bemühung um eine Publikation werden und würden wahrscheinlich auch nicht einfach in einer guten wissenschaftlichen Zeitschrift unterzubringen sein. 
Diese Überlegungen verdeutlichen, dass eine unselektionierte und flächendeckende Dokumentation der operativen Qualität notwendig ist, wenn grundsätzlich eine Information in dieser Hinsicht gewünscht wird und zu Zwecken der Qualitätssteuerung und -verbesserung genützt werden soll. Die Deutsche Gesellschaft für Gynäkologie und Geburtshilfe hat sich seit längerer Zeit um die Verwirklichung eines solchen Konzeptes bemüht und sich aktiv in Pilotprojekte eingebracht (s. Beiträge Köster und Berg). Bedauerlicherweise haben die bisherigen Bemühungen nicht zu dem Erfolg geführt, dass mit einer baldigen praktikablen Umsetzung zu rechnen ist.

Da mehrere Aktivitäten auf diesem Gebiet bestehen, muss klargestellt werden, welche Qualitätsmerkmale erfüllt sein müssen, um die wichtige Rolle einer verlässlichen Qualitätsdokumentation und gegebenenfalls die als Argumentationsbasis für Veränderungs-Überlegungen zu spielen. Dazu zählt im Einzelnen:

1. Die Qualität der Datenerhebung muss valide sein und angesichts der Bedeutung der sich aus ihr möglicherweise ergebenden weitreichenden Konsequenzen nicht infrage gestellt werden können.

2. Die Datenerhebung muss Indikations- und Risikomerkmale klar und zuverlässig einschließen.

3. Die Programme zur Datenerhebung sollten darauf hin ausgelegt sein, dass sie letztendlich eine offizielle Anerkennung erfahren und auf dieser Basis eine Finanzierung gesichert ist. Derzeit liegt die Verantwortung beim Bundeskuratorium.

4. Die sich aus der Mitwirkung von Ärztinnen und Ärzten vor Ort ergebenden Belastungen müssen definiert und abgeschätzt werden. Zwar sollte die Beurteilung von Indikations-Risiko und operativen Merkmalen letztendlich ärztliche Aufgabe bleiben. Auf der anderen Seite darf die Gesamtbelastung der ärztlichen Mitarbeiter mit Dokumentationsaufgaben ein vertretbares Maß nicht überschreiten, und ohne Kompensation dürfen nicht immer mehr administrative Arbeiten auf die ärztlichen Mitarbeiter konzentriert werden. Hier muss eine Unterstützung erfolgen, die sicherlich auch Rückwirkungen auf die Gesamtfinanzierung haben wird.

5. Die Vielschichtigkeit von Qualitäts-Überlegungen in der operativen Gynäkologie, wie sie in diesem Buch zum Ausdruck kommt und sicher noch um mehrere Aspekte zu erweitern wäre, muss möglichst frühzeitig und durchgehend berücksichtigt werden.
Dazu zählt auch der Umstand, dass zwischen der weiterentwickelten operativen Technik und der zur Abrechnung derselben herangezogenen Gebührenordnung für Ärzte in der Grundkonzeption aus den sechziger Jahren des letzten Jahrhunderts eine Kluft besteht. Daraus ergibt sich eine inakzeptable Diskrepanz zwischen dem, was heutzutage als state of the art gefordert und durchgeführt wird und dem, was formal dafür zu berechnen ist. Eine Arbeitsgruppe der Deutschen Gesellschaft für Gynäkologie und Geburtshilfe hat in Zusammenarbeit mit dem Berufsverband der Frauenärzte dazu Vorschläge für eine interimistische Lösung erarbeitet, da eine Novellierung der GOÄ, die dringend erforderlich wäre, auf absehbare Zeit nicht zu erwarten sein wird.

Wie immer man über die Form und die Folgen einer Qualitätskontrolle denkt, sie wird eine immer bedeutsamere Komponente unserer zukünftigen Tätigkeit sein. Statt über das „ob“ zu diskutieren, sollte man sich ganz auf das „wie“ konzentrieren. Nur so gibt es eine Möglichkeit, dass die Diskussion in erster Linie von Kompetenz gesteuert wird und nicht von fordernden Institutionen, für deren eigene Bewertung keine Qualitätsaussagen vorliegen oder angemahnt werden. 